\title{
Etched Optical Fiber Vibration Sensor to Monitor Health Condition of Beam Like Structures
}

\author{
Kishore PUTHA*, Dinakar DANTALA, \\ Srimannarayana KAMINENI, and Vengal Rao PACHAVA
}

National Institute of Technology, Warangal, A.P., India

*Corresponding author: Kishore PUTHA_ E-mail: kishorephd.nitw@gmail.com

\begin{abstract}
Using a center etched single mode optical fiber, a simple vibration senor is designed to monitor the vibrations of a simply supported beam. The sensor has high linear response to the axial displacement of about $0.8 \mathrm{~mm}$ with a sensitivity of $32 \mathrm{mV} / 10 \mu \mathrm{m}$ strain. The sensor is tested for periodic and suddenly released forces, and the results are found to coincide with the theoretical values. This simple design, small in size and low cost sensor may find applications in industry and civil engineering to monitor the vibrations of the beam structures and bridges.
\end{abstract}

Keywords: Optical fiber, etched fiber, simply supported beam, vibration sensor, health condition, monitoring

Citation: Kishore PUTHA, Dinakar DANTALA, Srimannarayana KAMINENI, and Vengal Rao PACHAVA, "Etched Optical Fiber Vibration Sensor to Monitor Health Condition of Beam Like Structures," Photonic Sensors, vol. 3, no. 2, pp. 124-130, 2013.

\section{Introduction}

Now-a-days structural beams are used in all branches of engineering and science, mainly for civil, mechanical, nuclear power plants and industrial structures. Simply supported beams are one of the important structures in real life. The dynamic vibration response of a beam with time and frequency gives enough information about the structural imperfections $[1,2]$. Generally, in real time, the forces are non-periodic and/or suddenly released forces [3]. The conventional structural health monitoring systems do not respond immediately to the damage, and also they are affected by electromagnetic waves. In contrast, fiber optic sensors are not affected by electromagnetic waves, and the material of the sensor is noncorrosive, moreover these are capable of measuring a variety of parameters such as the bending, displacement, vibration, temperature and pressure with the high sensitivity $[4,5]$. These sensors are stable even at high temperatures and harsh environments [6]. In general, fiber optic sensors are works based on interferometric, wavelength coding and intensity modulation. Among them, intensity modulated sensors are becoming more prominent in civil, mechanical, medical, and geometrical engineering due to their simple design, easy alignment and low $\operatorname{cost}[7,8]$.

In this paper, the design of an inexpensive fiber optic vibration sensor to monitor the dynamic response of the simply supported rectangular beam with the symmetric overhang is reported. The sensor works on the principle of intrinsic intensity modulation corresponding to a force applied at the center of the beam where the etched portion of the

Received: 22 November 2012 / Revised version: 10 January 2013

(C) The Author(s) 2013. This article is published with open access at Springerlink.com 
sensing fiber is attached. The system is tested for the applied and suddenly released forces. The sensor has advantages of the simple design, small size, flexibility in length and immunity to electromagnetic interference (EMI) which enables to replace the electronic sensors to avoid the electrical damage and are easily imbedded in composite structures.

\section{Theory of the simply supported beam}

To measure the vibration of a beam using the fiber optic vibration sensor considers a beam with a thickness " $h$ ", width " $b$ " and length " $l$ " supported by two rigid supports with the symmetric overhang " $m$ ". The geometry of the beam is shown in Fig. 1.

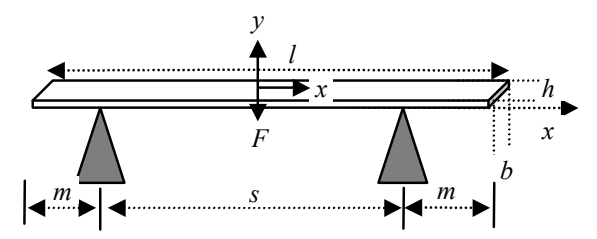

Fig. 1 Geometry of the simply supported beam.

As the dimensions of the cross-section are small in comparison to the length of the beam under consideration, the effect of shearing and rotatory deformation is ignored. Here, the transverse vibration of the beam is considered as a one-degree problem [9-11]. The lateral displacement of the beam along the length for the concentrated force or the load applied at the center is given by [12]

$$
\begin{gathered}
y=\frac{F x}{12 E I}\left(\frac{3 l^{2}}{4}-x^{2}\right) \text { for } 0<x<\frac{l}{2} \\
y=\frac{F}{12 E l}\left(x^{3}+\frac{9 x l^{2}}{4}-\frac{l^{3}}{4}-3 l x^{2}\right) \text { for } \frac{l}{2}<x<l
\end{gathered}
$$

where $E, F$ and $I$ are the moduli of elasticity of the spring-steel beam, force applied at the center of the beam and moment of inertia, respectively. The bending of the beam at the center where the force or load applied is

$$
y_{\max }=\frac{F}{4 E b}\left(\frac{l}{h}\right)^{3}
$$

The free transverse vibration of the simply supported beam corresponding to the concentrated load at the center is given by [13]

$$
y(x, t)=\sum_{n=1}^{\infty} \frac{2 F l^{3}}{n^{4} \pi^{4} E l} \sin \left(\frac{n \pi}{2}\right) \sin \left(\frac{n \pi x}{l}\right) \cos \left(w_{n} t\right)
$$

where $w_{n}$ is the natural frequency of the overhang beam and is given by

$$
w_{n}=\left(\frac{2 \pi}{s}\right)^{2} \sqrt{\frac{E l}{A \rho}}
$$

where $\rho$ and $A$ are the mass density and cross-sectional area of the beam, respectively.

When the force is applied at the center of the beam and released suddenly, the vibration of the beam undergoes a damped motion following a near step and giving the overshoot that slowly decays exponentially towards zero of the amplitude of the vibration due to the structural damping of the beam and is given by [14]

$$
\begin{aligned}
& y_{d}(x, t)= \\
& \sum_{n=1}^{\infty} \frac{2 F l^{3}}{n^{4} \pi^{4} E l} \sin \left(\frac{n \pi}{2}\right) \sin \left(\frac{n \pi x}{l}\right) \cos \left(w_{d} t\right) \exp \left(-\xi w_{n} t\right)
\end{aligned}
$$

where $w_{d}$ and $\xi$ are the damping frequency and the structural damping coefficient of the beam and are expressed as

$$
w_{d}=w_{n} \sqrt{1-\xi^{2}} \text { and } \xi=\frac{y_{\max }}{\sqrt{4 \pi^{2}+y_{\max }^{2}}} .
$$

\section{Etched fiber as a sensor}

Macro bending of the optical fiber can be sensed by measuring the intensity variation of the light through the fiber. One can reduce the diameter of the cladding by the chemical etching method or by D-shaping. Due to a reduction in the diameter of the cladding, the etched fiber is more sensitive to small changes in macro bending comparing to the unetched fiber response. When the fiber is stretched, the reduction in the cross-sectional area of the fiber results in a change in the refractive index. The 
change in the core radius and refractive index of the fiber leads to a change in the mode volume which is proportional to the strain [15]. When a small portion of the optical fiber is etched and the whole fiber is subjected to induced tensile macro-bending, at the etched and unetched junction, a mode volume mismatch occurs. This is due to an increase in the number of modes when light passes from the unetched portion to the etched portion of the fiber.

For an etched fiber, the refractive index of the cladding layer is higher than that of the surrounding air, and the whole fiber can be regarded as a multimode fiber because of the strong reflection and recoupling with the propagating light within the core [16]. When the etched fiber experiences a macro bending, due to the flexural strain, the mode volume mismatch between the etched and unetched portions of the fiber will cause a transmission power loss. The mode volume mismatch increases as the macro bending of the fiber increases, and the intensity of light sensed decreases within the limits of the bending. Among the two methods, to reduce the clad diameter of the fiber, chemical etching is well controllable, and it is an easy technique [16].

The single mode fiber (SMF-28) fiber of $9 / 125 \mu \mathrm{m}$ (core/cladding) is etched by the chemical method in a small region at the middle of the fiber. To etch the fiber, initially the protective plastic coating with a length of $1 \mathrm{~mm}$ is stripped off, and then it is immersed in the $40 \%$ hydrofluoric acid (HF) solution for a period of 30 minutes which results in a reduction in the fiber diameter about $50 \mu \mathrm{m}$. The etching is monitored in real time by measuring the power of the transmitted light coming from a broad band source coupled through a circulator to the fiber Bragg grating (FBG) and coupling the reflected light from the FBG to the sensing fiber. The etching process is terminated when the power reduces rapidly due to the hydrofluoric acid entering the evanescent field region.

Once the etching process is terminated, the fiber is cleaned with the distilled water and ethanol to remove the residues of $\mathrm{HF}$ and then dried under vacuum condition [17, 18]. Figure 2 shows the optical microscope photograph of the unetched and etched parts of the fiber.

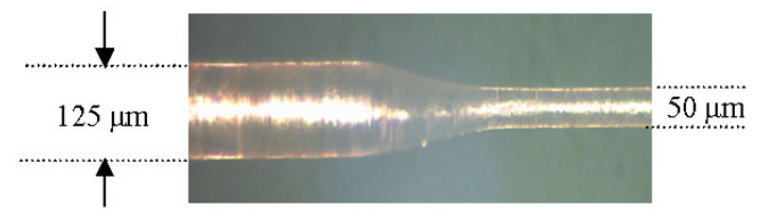

Fig. 2 Unetched and etched parts of the fiber.

\section{Experiment}

The schematic experimental setup is shown in Fig. 3. It consists of a spring-steel beam having Young's modulus $(E)$ of $200 \mathrm{MPa}$, the moment of inertia in area $(I)$ of $260.4167 \mathrm{~mm}^{4}$, the material mass density $(\rho)$ of $7850 \mathrm{~kg} / \mathrm{m}^{3}$ with dimensions, $l=308 \mathrm{~mm}, b=25 \mathrm{~mm}$ and $h=0.5 \mathrm{~mm}$ and supported on two rigid bases with the overhang $(\mathrm{m})$ with a length of $64 \mathrm{~mm}$. Light from a broad band source (OEFSS-100) with a center wavelength of $1550 \mathrm{~nm}$ is coupled to an FBG of the Bragg wavelength at $1540.32 \mathrm{~nm}$ through a circulator at the room temperature. A matched fiber pigtailed photo-detector with a typical responsivity of $0.9 \mathrm{~A} / \mathrm{W}$ at $1550 \mathrm{~nm}$ has the saturation power of $2 \mathrm{~mW}$ used along with the trans-impedance amplifier circuitry to convert the light intensity into its equivalent voltage signal. A data acquisition system (NI DAQ - 6016) having the maximum sampling frequency of $200 \mathrm{kHz}$ with a 8 -bit resolution is used to record the voltage signal acquired from the transimpedance amplifier at the sampling rate of $2 \mathrm{kHz}$ and monitor the vibration of the beam. The etched portion of the fiber with a length of $1 \mathrm{~mm}$ is glued at the center of the beam with an adhesive. The light is coupled to the node 1 of the circulator and directed to the FBG through the node 2 of the 
circulator. The reflected peak of the FBG is coupled to the etched fiber through the node 3 of the circulator. The fiber end of the FBG is cleaved, and an index matching gel (IMG) is used to minimize the noise level and reflection from the end of the fiber.

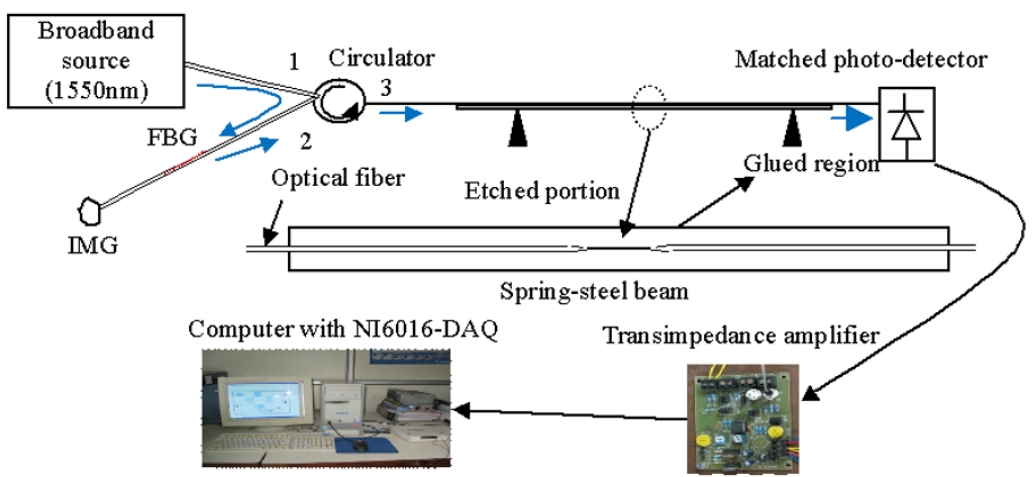

Fig. 3 Schematic of the experimental setup.

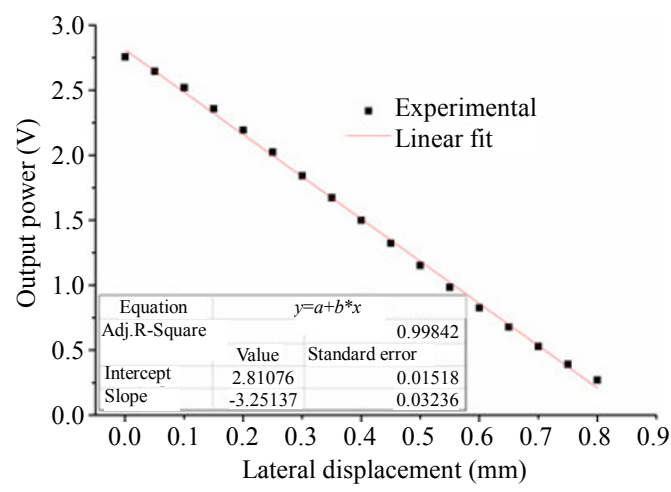

Fig. 4 Response of the sensor for the lateral displacement at the center of the beam.

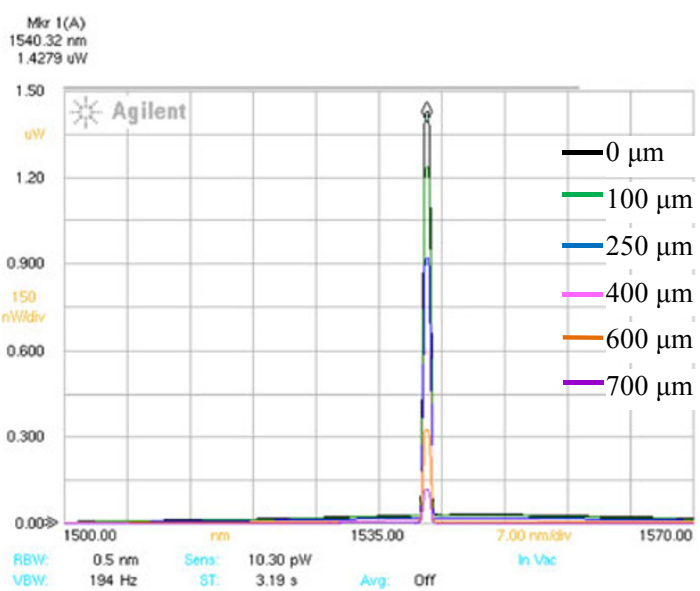

Fig. 5 Optical spectral response of the sensor for different lateral displacements of the beam.

To calibrate the sensor, the load is applied at the center of the spring-steel beam in terms of the bending where the etched fiber is attached. Using a precise micro meter, lateral bending of the beam in steps of $50 \mu \mathrm{m}$ is achieved. The measured the response of the sensor is plotted in Fig. 4. It is from Fig. 4 that the sensor has a high linear response for a lateral displacement of $0.8 \mathrm{~mm}$ and a sensitivity of $32 \mathrm{mV} / 10 \mu \mathrm{m}$ strain. The spectral response of the sensor for different lateral displacements is also shown in Fig. 5.

\section{Results and discussions}

Figure 6 illustrates the simulation results for the

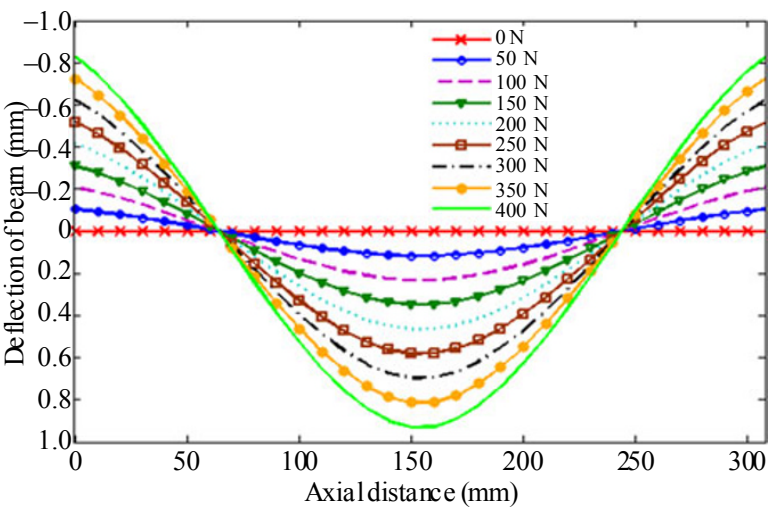

Fig. 6 Lateral deflection of the beam axially.

lateral bending of the beam along the length for different forces applied at the center of the beam using (1) and (2). By applying the varying loads at the center of the beam, the bending of the beam for different forces at that point is plotted for theoretical 
results using (4), and experimental results is shown in Fig. 7. From Fig. 7, it is clear that the bending response for the applied force is linear, and both theoretical and experimental results are in good agreement. The discrepancy between theoretical and experimental results may be attributed to the fact that the values of the parameters used for the simulation may not be exact values, ignorance of ambient conditions and also neglectful of some parameters to reduce the complexity for simplifying the equation.

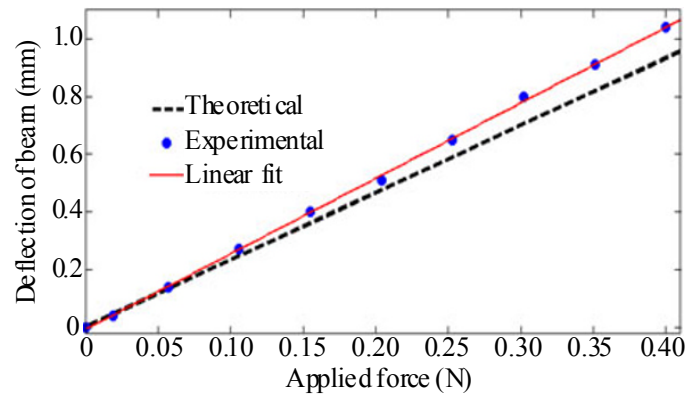

Fig. 7 Theoretical and experimental responses of the beam bending for the applied force.

To test the sensor, the beam is subjected to bending periodically using the 8051 stepper motor with a cam of the offset $1 \mathrm{~mm}$ at a speed of $6 \mathrm{rpm}$, and the response of the sensor is recorded using a data acquisition system (Fig. 8). The offset portion in

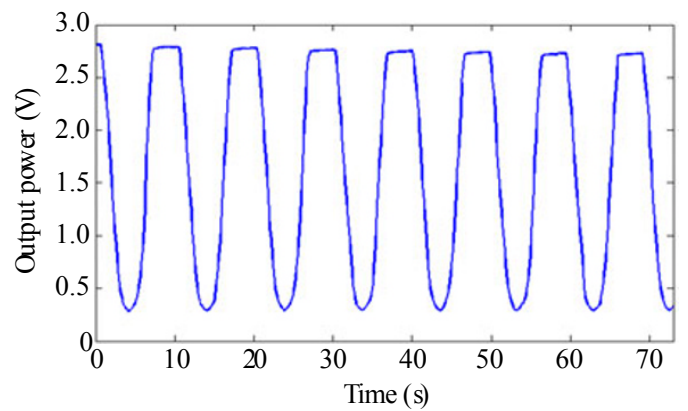

Fig. 8 Response of the sensor for the periodic vibration of the beam.

Fig. 8 shows the stability of the signal. The sensor is also tested for the vibrations of the beam for the force applied at the center and then released suddenly; the response of the sensor is shown in Fig. 9. The exponential decay of the amplitude of the vibration of the beam after releasing the force is in good agreement with the theoretical response of the damped motion from (6). The fast Fourier transform (FFT) spectrum of the damped motion of the beam for an applied short range force of $0.3 \mathrm{~N}$ is shown in Fig. 9 and observed the peak frequency of the vibration nearly at $17 \mathrm{~Hz}$.

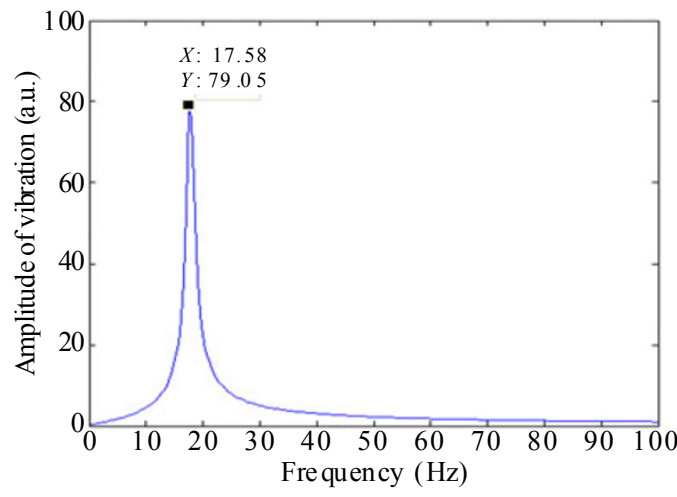

Fig. 9 FFT of the damped vibration of the beam.

In Fig. 10, the curve between points $a$ to $b$ is the response of the beam to the near step force, and then it overshoots to under the damped motion due to the structural damping and slowly comes to the zero amplitude of the vibration with time. The FFT of the time domain signal gives the frequency of the vibration of the beam and is found to be $17 \mathrm{~Hz}$, which is nearly matched with the obtained theoretical value.

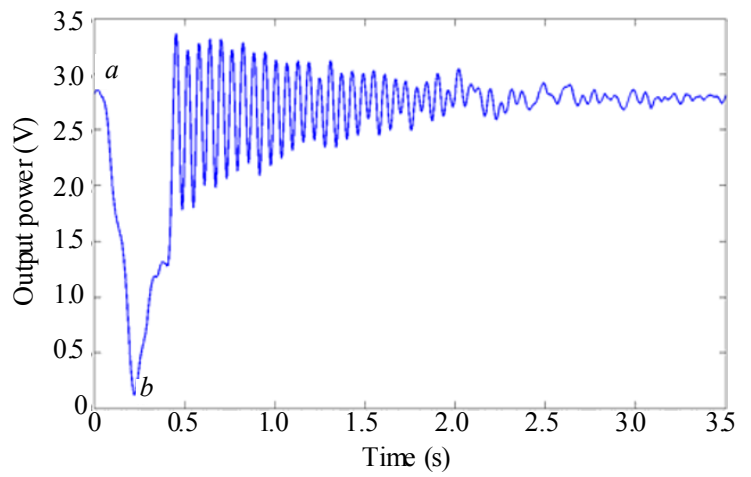

Fig. 10 Response of the sensor for the suddenly released force at the center of the beam.

The time and frequency responses of the beam give the sufficient information to know the health condition of the beam. The sensor is also tested for 
the suddenly released step force applied repeatedly, and the corresponding response is shown in Fig. 11. As the length of the fiber (unetched) of the sensor plays an insignificant role in the power loss, it is recommended for real-time health monitoring systems. When compared with the well know FBG sensing system, it has the advantage of the high sensitivity, easy maintenance, low cost and simpleness in design. The sensitivity of the sensor can be changed by altering the diameter and/or length of the etched portion of the fiber. This sensor may not be suitable for inline sensing but can be used for distributed sensing.

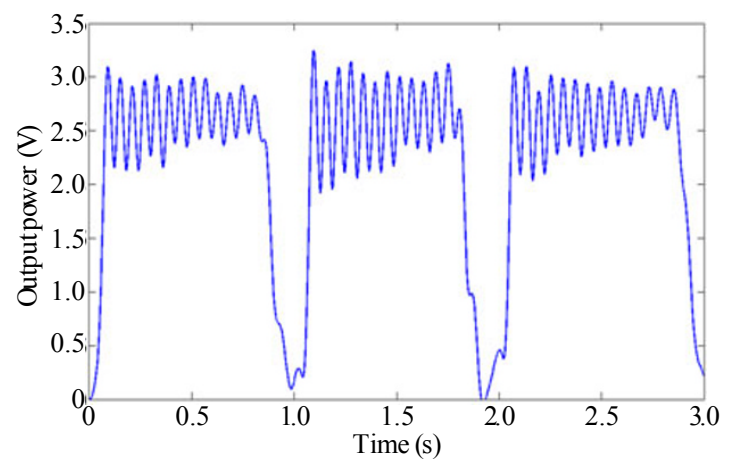

Fig. 11 Response of the sensor for repeatedly applied force to the beam.

\section{Conclusions}

An attempt to design a simple fiber optic vibration sensor to monitor the health condition of simply supported beam which is symmetrically over-hanged is discussed. The theoretical and experimental responses are in good agreement. The sensor shows a high linear response of the $0.8 \mathrm{~mm}$ lateral displacement with a sensitivity of the $32 \mathrm{mV} / 10 \mu \mathrm{m}$ strain. The sensor is tested for forced vibrations of the beam such as suddenly released forces and periodic forces, which would be more realistic and appropriate for the real field. The temporal response of the beam is recorded, and the frequency response gives enough information about the health condition of the beam. The sensor has its own advantages such as the simple design, economy, flexibility in length and smallness in size, enables for remote monitoring of the beam like structures.

Open Access This article is distributed under the terms of the Creative Commons Attribution License which permits any use, distribution, and reproduction in any medium, provided the original author(s) and source are credited.

\section{References}

[1] J. D. Yau, "Vibration of simply supported compound beams to moving loads," Journal of Marine Science and Technology, vol. 12, no. 4, pp. 319-328, 2004.

[2] D. Wang, T. Li, and Z. Yuan, "The simulated analysis based on EMD and shape factor to identify damage location of a simple supported beam steel bridge," in International Conference on Digital Manufacturing \& Automation, Qinhuangdao, China, Dec. 18-20, pp. 808-810, 2010.

[3] L. Meirovitch, Elements of vibration analysis, Second edition. New York, USA: McGraw-Hill, 1986.

[4] K. S. C. Kuang, W. J. Cantwell, and P. J. Scully, “An evaluation of a novel plastic optical fiber sensor for axial strain and bend measurements," Measurement Science and Technology, vol. 13, no. 10, pp. 1523-1534, 2002.

[5] C. H. Chen, Y. L. Shen, and C. S. Shin, "Using distributed Brillouin fiber sensor to detect the strain and cracks of steel structures," Journal of Mechanics, vol. 26, no. 4, pp. 547-551, 2010.

[6] M. Zhang, X. Ma, L. Wang, S. Lai, H. Zhou, H. Zhao, et al., "Progress of optical fiber sensors and its application in harsh environment," Photonic Sensors, vol. 1, no. 1, pp. 84-89, 2011.

[7] W. R. Habel and K. Krebber, "Fiber-optic sensor applications in civil and geotechnical engineering," Photonic Sensors, vol. 1, no. 3, pp. 268-280, 2011.

[8] P. Roriz, A. Ramos, J. L. Santos, and J. A. Simoes, "Fiber optic intensity-modulated sensors: a review in biomechanics," Photonic Sensors, vol. 2, no. 4, pp. 315-330, 2012.

[9] J. F. Murphy, “Transverse vibration of a simply supported beam with symmetric overhang of arbitrary length," Journal of Testing and Evaluation, vol. 25, no. 5, pp. 552-524, 1997.

[10] S. M. J. Ali and Z. S. Al-Sarraf, "Study the 
transverse vibration of beam with different length," Al-Rafidain Engineering Journal, vol. 17, no. 1, pp. 83-91, 2009.

[11] S. Timoshenko and D. H. Young, Vibration problems in engineering, Third edition. New Delhi: East-West Press Pvt. Ltd., 1964.

[12] W. A. Nash, Schaum's outline of theory and problems of strength of materials, Fourth edition. New York, USA: McGraw-Hill, 1998.

[13] S. M. Abdullah, "Free vibration of simply supported beams using Fourier series," Al-Rafidain Engineering Journal, vol. 14, no. 2, pp. 51-67, 2006.

[14] S. G. Kelly, Fundamentals of mechanical vibrations, International editions. New York, USA: McGraw-Hill, 1993.

[15] M. Vaziri and C. L. Chen, "Etched fibers as strain gauges," Journal of Lightwave Technology, vol. 10. no. 6, pp. 836-841, 1992.

[16] Y. Zaatar, D. Zaouk, J. Bechara, A. Khoury, C. Llinaress, and J. P. Charles, "Fabrication and characterization of an evanescent wave fiber optic sensor for air pollution control," Materials Science and Engineering: B, vol. 74, no. 1, pp. 296-298, 2000.

[17] X. Tian, X. Cheng, W. Qiu, Y. Luo, Q. Zhang, B. Zhu, et al., "Optically tunable polarization state of propagating light at $1550 \mathrm{~nm}$ in an etched single-mode fiber with azo-polymer overlay," IEEE Photonics Technology Letters, vol. 23, no. 3, pp. 170-172, 2011.

[18] P. Wang, Q. Wang, G. Farrell, G. Rajan, T. Frier, and J. Cassidy, "Investigation of macro bending losses of standard single mode fiber with small bend radii," Microwave and Optical Technology Letters, vol. 49, no. 9, pp. 2133-2138, 2007. 